\title{
Patterns of Admission Cardiotocography Decisions and Associated Perinatal Outcomes in a Southern Nigerian Teaching Hospital
}

\author{
J. O. Imaralu ${ }^{1^{\star}}$, F. I. Ani ${ }^{1}$, A. O. Olaleye ${ }^{1}$, J. O. Sotunsa ${ }^{1}$, A. Akadri $^{1}$ \\ and O. O. Adebawojo 1 \\ ${ }^{1}$ Department of Obstetrics and Gynaecology, Babcock University Teaching Hospital, Ilishan-Remo, \\ Nigeria.
}

Authors' contributions

This work was carried out in collaboration among all authors. Author JOI conceptualized and together with author FIA designed the study, including the study protocol. Authors AA, JOI and OOA managed the literature search and data acquisition. The statistical analysis and first draft of the manuscript was done by authors $\mathrm{JOI}$ and $\mathrm{AOO}$, with substantial contributions from authors AA and JOS. All authors read and approved the final manuscript.

Article Information

DOI: 10.9734/ARRB/2019/v33i230117

Editor(s):

(1) Dr. Moacir Marocolo, Department of Physiology, Physiology and Human Performance Research Group, Federal University of Juiz de Fora, Brazil.

Reviewers:

(1) Joel Noutakdie Tochie, University of Yaounde 1, Cameroon (2) Alexandrina Cardoso, Escola Superior de Enfermagem do Porto, Portugal.

(3) Reda Mohamed Nabil Aboushady, Cairo University, Egypt. Complete Peer review History: http://www.sdiarticle4.com/review-history/51777

Original Research Article

Received 26 July 2019

Accepted 30 September 2019

Published 12 October 2019

\section{ABSTRACT}

Aims: To determine the patterns of obstetric decisions based on Cardiotocography (CTG) findings and the effect of these decisions on labour outcomes.

Study Design: Retrospective observational audit.

Place and Duration of Study: Department of Obstetrics and Gynaecology, Babcock University Teaching Hospital, Ilishan-Remo. Data were obtained from all intrapartum CTG tracings done from January 2016 to December 2018.

Methodology: A consecutive sampling technique was used and the case files, delivery register and follow up records of all included CTG tracings were used to obtain maternal and perinatal data. Data were analyzed using the SPSS version 21.0. Numerical data were expressed as mean \pm standard 
deviation (SD). The Chi-square test was used to compare categorical variables while the independent T-test was used to compare means of groups studied. Binary logistic regression was used to assess the factors related to maternal and perinatal outcomes. The level of statistical significance was be set at $p$-value of $<0.05$.

Results: There were 756 deliveries, but only 436 CTGs met the inclusion criteria. The prevalence of abnormal CTG was $130 / 436(29.8 \%) ; 93 / 436(21.3 \%)$ were suspicious while $37 / 436(8.5 \%)$ were pathological. On the basis of CTG; $300 / 436$ (68.8\%) of the parturients had intermittent fetal heart tone auscultation, 100/436 (22.9\%) had continuous Electronic fetal monitoring (EFM) while labour was stopped in $36 / 436(8.3 \%)$. The $5^{\text {th }}$ minute APGAR score did not depend on the admission CTG finding (0.55), the decision to stop or continue labour $(p=0.26)$ or the use of continuous EFM $(P=0.66)$. Maternal near miss (MNM) was not a consequence of the decision to stop labour $(P=0.98)$ or the use of continuous EFM $(P=0.19)$. The mode of delivery outcome was however a consequence of decisions to continue or stop labour $(P<0.001, \mathrm{AOR}=0.202,95 \% \mathrm{Cl}=0.153-0.265)$. Pregnancy risk was a consistent determinant of maternal outcomes; occurrence of MNM $(P<0.001$, $\mathrm{AOR}=0.002,95 \% \mathrm{Cl}=0.000-0.032)$ and mode of delivery $(P<0.001, \mathrm{AOR}=0.015,95 \% \mathrm{Cl}=0.005-$ 0.043).

Conclusion: Decisions based on admission CTG was associated with a high rate of operative deliveries, without any significant effect on fetal or maternal outcomes. CTG in labour should be restricted to pregnancies adjudged as high risk based on obstetric factors and CTG should be used as an adjunctive triaging instrument.

Keywords: Admission test; asphyxia; cardiotocography; caesarean section; fetal monitoring.

\section{INTRODUCTION}

Intrapartum assessment of the parturient in labour has been done with a two way communication through the ages and presence of danger signs easily detected through physical contact with the expectant mothers taking advantage of the fact that these adults can talk and express their perceptions and discomfort about the labour process. This is not so with the passenger- the fetus, whom the attendant cannot see or hear. Fetal surveillance in labour has been shown in research reports to be very important to ensure the delivery of a healthy baby [1]. Intrapartum fetal monitoring has evolved from perception of fetal movements through intermittent auscultation with the fetal stethoscope to cardiotocography which has been proven to be useful and is today the most widely recommended indirect diagnostic procedure for fetal monitoring during pregnancy and labour [1]. ${ }^{1}$ The role of intermittent fetal heart rate monitoring either with the fetal stethoscope or the Doppler hand held ultrasound aid in labour is well established and accepted, however routine use of cardiotocography for all parturients remains debatable because of linkage to high rates of operative interventions with little or no maternal or perinatal benefits [2].

The admission CTG test is described as a short recording of the $\mathrm{FHR}$ and uterine contraction patterns during labour by the use of the CTG device for 20-30 minutes upon admission to the labour room [3]. Labour is known to be associated with some stress from uterine contractions with potential to cause acidosis to some foetuses classified as low-risk. While it is recommended that admission cardiotocography be reserved for high risk labour, because it was shown to be highly effective in detecting fetal compromise and preventing adverse outcome in the babies of high risk parturient, other researchers have suggested admission cardiotocography as the actual triage for the risk of fetal distress in labour rather than the admitting diagnosis as a significant number of low risk parturient with abnormal admission CTG later developed fetal distress [4].

Two major factors would drive policy formulation regarding antepartum and intrapartum fetal monitoring in contemporary obstetrics practice. These include the unacceptably high perinatal mortality and morbidity rates in the low and middle income countries and the ever increasing awareness of potential litigation in the developed countries of the world. Admission CTG is routine in many centres today including our own, but can this practice be justified? This retrospective observational study which also serves as a practice audit is therefore aimed to determine the incidence of CTG abnormalities in labour; the nature of decisions taken based on these abnormalities and the immediate perinatal outcomes associated with these decisions in a 
private owned teaching hospital in southern Nigeria.

\section{MATERIALS AND METHODS}

This study was conducted in the department of Obstetrics and Gynaecology of the Babcock University Teaching Hospital (BUTH), IlishanRemo, Nigeria. The BUTH hospital records from 2012; reveals that annually an average of 260 deliveries occurs at BUTH.

\subsection{Study Design}

Retrospective observational study.

\subsection{Study Location}

The department of Obstetrics and Gynaecology, of the Babcock University Teaching Hospital, a private-sector owned tertiary level centre in Ilishan-Remo, south western Nigeria.

\subsection{Study Duration}

6 months of data extraction and analysis.

\subsection{Sample Size and Sampling Technique}

A consecutive sampling technique was applied and all external CTG tracings done within the 3year period 2016-2018 were included in the analysis.

\subsection{Subjects and Selection Method}

Data were obtained from all CTG tracings done in the labour ward units of BUTH that met the inclusion criteria.

\subsection{Inclusion Criteria}

1. All external CTG tracings of patients admitted in labour.

2. Gestational age between 38 and 41 completed weeks.

3. Singleton pregnancies.

\subsection{Exclusion Criteria}

1. Obstetric emergency requiring immediate delivery

2. Preterm labour

3. Induction of labour

4. Multiple gestation

5. Intrauterine fetal death (IUFD)
6. Maternal cardiac disease or medication for cardiac disease

7. Congenitally abnormal fetus earlier detected by ultrasound scanning

8. Advanced labour (cervical dilatation of $8 \mathrm{~cm}$ or more) or delivery within 2 hours of admission

\subsection{Procedure}

Data extraction was done by a team of three investigators of senior registrar/consultant level using a proforma, designed for this purpose. The NICE management based CTG diagnostic criteria were used to make a research CTG diagnosis [5]. The extracted data were then entered into the analysis software by a team of research assistants. Both data extraction and entry lasted for a period of 6 months.

CTG at BUTH within the period 2016-2018 was done in all cases using the EDAN F6 fetal and maternal monitor version 1.5 [EDAN instruments Inc. USA 2008-2012]. ${ }^{\odot}$ The machine speed was set to default paper speed of $3 \mathrm{~cm} /$ minute and a vertical height of $20 \mathrm{bpm}$ throughout. All CTG tracings were routinely reviewed by the consultant/senior registrar and decisions ranging from extension or repeat of the CTG, continuation of labour with continuous electronic fetal monitoring, intermittent auscultation with the fetal stethoscope to stopping labour and immediate delivery were all based on the findings of the CTG.

A proforma was used to extract the obstetric biodata of the parturient obtained from the hospital case records including records of vital signs and admission diagnosis. The summary of the outcome of the labour process was obtained from the partograph, the operation or delivery notes and other records of the parturients in the hospital.

The perinatal outcome variables were APGAR scores at 1 and 5 minutes, meconium passage, fetal distress, intrapartum fetal death and early neonatal death.

The maternal outcome variables were maternal near miss, maternal death, occurrence of maternal complication of labour and mode of delivery.

Decisions were defined to include all activities done from extension of the CTG to intrapartum continuous electronic fetal monitoring (EFM), to 
delivery decisions like Caesarean Section (CS) or other operative vaginal delivery.

Operative delivery was defined to include caesarean section, vacuum or forceps delivery.

High risk pregnancy was defined as one with a risk of adverse maternal or fetal outcome greater than the general obstetric population. Admission diagnosis was thus classified, based on the risk of adverse labour outcome, as one in which the risk of adverse maternal or perinatal outcome of labour is higher than the general obstetric population and these outcomes included; fetal distress, intrapartum fetal demise, low APGAR scores and early neonatal death, maternal near miss, maternal death and the risk of operative delivery, while low risk pregnancy was defined as one in which the risk of adverse maternal or perinatal outcome was lower than in the general obstetric population [6].

Active phase of labour was defined as cervical dilatation finding of $4 \mathrm{~cm}$ or greater and at least three uterine contractions within 10 minutes, each lasting more than 35 seconds, while latent phase of labour was defined as the finding of 2 or more uterine contractions that lasted more than 40 seconds at term, irrespective of cervical dilatation.

Maternal complication of labour was defined as complications that occurred to the woman only because of the labour process and this includes; slow progress in labour, prolonged labour, cephalopelvic disproportion, ruptured uterus, cervical laceration, postpartum haemorrhage, uterine inversion, retained placenta, amniotic fluid embolism, etc.

Maternal Near Miss (MNM) was defined as an acute obstetric complication that immediately threatened a woman's survival but did not result in her death by chance or because of hospital care she received during labour or immediately postpartum [7].

\subsection{Statistical Analysis}

Data were analyzed using the SPSS version 21.0 (SPSS Inc., Chicago, IL). Numerical data were expressed as mean \pm standard deviation (SD). Bivariate analysis was done and the independent T-test used to compare of means of groups studied, while the Chi-square test was used to compare categorical variables. Binary logistic regression was used to assess the factors related to the maternal and perinatal outcomes. The level of statistical significance was set at $p$-value of $<0.05$.

\section{RESULTS}

There were a total of 756 deliveries within the period covered by this audit; however only 436 labour admissions met the inclusion criteria. The others were 149 elective caesarean sections, 144 obstetric complications that had emergency deliveries and 27 parturients that presented in advanced labour.

The median age of the parturients was 30 years (range 18-42), most 232/436 (53.2\%) of the women had secondary level education, were employed 374/436 (85.8\%), married 427/436 $(97.9 \%)$ and lived within $5 \mathrm{~km}$ distance from the hospital 299/436 (68.6\%). Although, the parturient were mostly nulliparous, 222/436 $(50.9 \%)$, the median interval from the last child birth among the women who had delivered before was 2 years (range 1-8). All of the parturients were booked for ANC at the study centre. The prevalence of high risk pregnancy was $82 / 436(18.8 \%)$ and $13 / 436(3 \%)$ of the women had a history of previous caesarean section. The mean gestational age at presentation was $38.7 \pm 1.3$ weeks (Table 1).

Table 2 outlines the decisions taken after admission CTG for intrapartum FHR monitoring, 275/436 (63.1\%) of the parturient were allowed to continue labour with intermittent fetal heart tone auscultation with the Pinard's stethoscope while 98/436 (22.5\%) had continuous EFM. The others; 28/436 (6.4\%) had repeat CTG or extended CTG beyond 20 minutes and 35/436 (8.0\%) had labour stopped for immediate delivery. After extension or repeat CTG, $300 / 436(68.8 \%)$ of the parturients had their labour managed with intermittent fetal heart tone auscultation while 100/436 (22.9\%) had continuous EFM, 36/436 $(8.3 \%)$ had labour stopped for immediate delivery. The heterogeneous interpretation of the CTG findings led to interpretation by the clinicians, using the NICE management based-guidelines, and the observations made include: normal CTG finding in 306/436 (70.2\%) of the parturient, suspicious CTG tracing in 93/436(21.3\%) while $37 / 436 \quad(8.5 \%)$ had pathological CTG tracings. Thus an incidence of $130 / 436 \quad(29.8 \%)$ was obtained for abnormal (suspicious or pathological) CTG finding. 
Table 1. Socio-demographic characteristics of the parturient

\begin{tabular}{|c|c|c|}
\hline Parameter & Frequency $(n=436)$ & Percentage (\%) \\
\hline Age [median(range)] & $30.0(18-42)$ years & \\
\hline \multicolumn{3}{|l|}{ Educational level } \\
\hline Primary & 7 & 1.6 \\
\hline Secondary & 232 & 53.2 \\
\hline Tertiary & 197 & 45.2 \\
\hline \multicolumn{3}{|l|}{ Occupation } \\
\hline Unemployed & 62 & 14.2 \\
\hline Employed & 374 & 85.8 \\
\hline \multicolumn{3}{|l|}{ Marital status } \\
\hline Single & 9 & 2.1 \\
\hline Married & 427 & 97.9 \\
\hline \multicolumn{3}{|l|}{ Residence from hospital } \\
\hline$<5 \mathrm{~km}$ & 299 & 68.6 \\
\hline$>5 \mathrm{~km}$ & 137 & 31.4 \\
\hline \multicolumn{3}{|l|}{ ANC Booking status } \\
\hline Unbooked & 0 & 0 \\
\hline Booked & 436 & 100 \\
\hline \multicolumn{3}{|l|}{ Parity } \\
\hline 0 & 222 & 50.9 \\
\hline$\geq 1$ & 214 & 49.1 \\
\hline No. of children alive [median(range)], $n=214$ & $0(0-5)$ & \\
\hline Last child birth [median(range)] & $2(1-8)$ years ago & \\
\hline \multicolumn{3}{|l|}{ Previous CS } \\
\hline No & 423 & 97 \\
\hline Yes & 13 & 3 \\
\hline Gestational age (mean) & $38.7 \pm 1.3$ weeks & \\
\hline \multicolumn{3}{|l|}{ Pregnancy risk } \\
\hline Low risk pregnancy/labour & 354 & 81.2 \\
\hline High risk pregnancy/Labour & 82 & 18.8 \\
\hline \multicolumn{3}{|l|}{ Phase of labour at admission } \\
\hline Latent phase & 68 & 15.6 \\
\hline Active phase & 368 & 84.4 \\
\hline
\end{tabular}

Table 3 highlights the determinants of admission CTG-based decisions for FHR monitoring during labour; only the CTG findings had a statistically significant relationship with the decision to stop or continue labour $(p<0.001, \quad O R=0.025$, $95 \% \mathrm{Cl}=0.006-0.102)$. The socio-demographic factors; age $(p=0.61)$, tertiary education $(p=0.30)$, paid employment $(p=0.80)$, parity $(0.30)$, previous caesarean section $(p=1.000)$, BMI $(p=0.17)$, maternal pulse rate $(0.60)$, temperature $(p=0.72)$, stage of labour $(p=0.63)$ pregnancy risk $(p=1.00)$ and fetal presentation $(p=1.00)$ did not have any determining effect on decisions that were taken after admission CTG.

Table 4 highlights the effect of the admission CTG-based decisions on perinatal outcomes. The occurrence of fifth minute APGAR score $\leq 7$ had no statistically significant relationship with the decision to stop labour $(p=0.26)$, the intrapartum FHR monitoring pattern $(p=0.66)$ or the admission CTG finding (0.55). Similarly, occurrence of fetal distress in labour was not significantly determined by the pattern of intrapartum FHR monitoring $(p=0.42)$ or the admission CTG finding $(p=0.91)$. There was no intrapartum fetal death or early neonatal death in the parturient that were included in this study.

Table 5 also reveals that occurrence of maternal near miss (MNM) was not a consequence of the decision to stop labour $(p=0.98)$ or the pattern of intrapartum FHR monitoring $(p=0.19)$ neither did it depend on the CTG finding at admission $(p=0.38)$. This table also shows that intrapartum FHR monitoring pattern $(p=0.34)$ and admission CTG findings $(p=0.38)$ were not significant determinants of occurrence of maternal complications of labour. Pregnancy risk was however a significant determinant of occurrence 
Table 2. The patterns of decisions for Fetal Heart Rate (FHR) monitoring during labour and CTG findings

\begin{tabular}{|c|c|c|c|}
\hline \multicolumn{4}{|c|}{ Patterns of decisions for FHR monitoring during labour, based on initial admission CTC } \\
\hline Specific decision & \multicolumn{2}{|c|}{ Frequency } & Percentage \\
\hline Extend/repeat CTG & \multicolumn{2}{|l|}{28} & 6.4 \\
\hline Continue labour with continuous EFM & \multicolumn{2}{|l|}{98} & 22.5 \\
\hline Continue labour with intermittent auscultation & \multicolumn{2}{|l|}{275} & 63.1 \\
\hline Stop labour/immediate delivery & \multicolumn{2}{|l|}{35} & 8.0 \\
\hline Total & 436 & & 100 \\
\hline \multicolumn{4}{|c|}{ Final management decisions for FHR monitoring after repeat or extended CTG } \\
\hline Final CTG-based decision & Frequency & & Percentage \\
\hline Continue labour with continuous EFM & 100 & & 22.9 \\
\hline \multirow{2}{*}{ Continue labour with intermittent auscultation } & 300 & & 68.8 \\
\hline & 36 & & 8.3 \\
\hline Total & 436 & & 100 \\
\hline \multicolumn{4}{|c|}{ Classes of research interpreted admission CTG findings } \\
\hline \multirow[t]{3}{*}{ Phase of labour } & \multicolumn{3}{|c|}{ Management based CTG diagnosis } \\
\hline & \multicolumn{2}{|c|}{ Abnormal } & \multirow[t]{2}{*}{ Total } \\
\hline & Suspicious & Pathological & \\
\hline Latent phase & 12 & 7 & 68 \\
\hline Active phase & 81 & 30 & 368 \\
\hline $306(70.2 \%)$ & $93(21.3 \%)$ & $37(8.5 \%)$ & 436 \\
\hline
\end{tabular}

of MNM ( $p<0.001, A O R=0.002,95 \% C l=0.000$ 0.032 ) and occurrence of maternal complications of labour $(p<0.001, A O R=0.142,95 \% \mathrm{Cl}=0.069$ 0.292).

Table 6 however highlights the observation of a statistically significant relationship between the decision to continue labour and lower incidence of operative deliveries $(p<0.001, A O R=0.202$, $95 \% \mathrm{Cl}=0.153-0.265)$. It also shows that intermittent auscultation with the Pinard's stethoscope was comparable with continuous EFM $(p=0.22)$, with respect to mode of delivery outcome. Pregnancy risk was observed to be a significant determinant of mode of delivery as women with low risk pregnancies had significantly less risk of operative deliveries $(p<0.001, A O R=0.015,95 \% \mathrm{Cl}=0.005-0.043)$.

\section{DISCUSSION}

This audit was aimed to determine the prevalence of CTG abnormalities, the management decisions based on them and the effect of these decisions on perinatal and maternal outcomes of labour. A significant proportion of parturient that had an abnormal CTG finding were made to undergo immediate operative delivery, although these decisions did not result in significant immediate benefit to the babies.

The proportion of women who had their labour stopped because of a CTG abnormality is comparable to findings from a study that used admission CTG as screening test [8]. The use of the admission CTG test as triage in labour has continually been a subject of debate, with varying conclusions from reports. While proponents allude to its effect in improving neonatal outcomes, guidelines based on findings from systematic reviews have continually highlighted its potential to increase operative delivery rates, with added risk to the mothers without significant improvements in perinatal survival. Decisions or inactions from CTG managed labours have however been the bane of many obstetricians that were enmeshed in labour-related litigations [4,8-12].

CTG remains in use and several authors have blamed wrong or non-uniform interpretation of the findings for the disparity in analysis and decisions, even amongst experienced obstetricians $[13,14]$. We observed heterogeneous interpretations of the CTG tracings which led to a re-classification for the purpose of this audit, using the NICE management based-guidelines [5]. In many cases, the terminology 'abnormal' was used, but the specific abnormality was not stated, other terms used to arrive at decisions included, 'reassuring or non-reassuring'. In other instances, a management based diagnosis was not made and terminology like 'persistent tachycardia' or 'bradycardia', were found in the case records. 
Table 3. Determinants of admission CTG-based decision to continue FHR monitoring or stop labour

\begin{tabular}{|c|c|c|c|c|c|}
\hline \multirow[t]{2}{*}{ Factor } & \multicolumn{2}{|c|}{ CTG-based decision } & \multicolumn{2}{|c|}{$95 \% \mathrm{Cl}$} & \multirow[t]{2}{*}{$P$-value } \\
\hline & $\begin{array}{l}\text { Intermittent } \\
\text { auscultation/EFM } \\
(n=400)\end{array}$ & $\begin{array}{l}\text { Stop } \\
\text { labour/immediate } \\
\text { delivery }(n=36)\end{array}$ & Lower & Upper & \\
\hline Age (years) & $30.2 \pm 4.7$ & $30.6 \pm 4.1$ & -2.00 & 1.18 & 0.61 \\
\hline \multicolumn{6}{|l|}{ Tertiary education } \\
\hline No $(n=239)$ & $216(90.4 \%)$ & $23(9.6 \%)$ & & & 0.30 \\
\hline Yes $(n=197)$ & $184(93.4 \%)$ & $13(6.6 \%)$ & 0.76 & 2.80 & $\mathrm{OR}=1.46$ \\
\hline \multicolumn{6}{|l|}{ Paid employment } \\
\hline No $(n=62)$ & $56(90.3 \%)$ & $6(9.7 \%)$ & & & 0.80 \\
\hline Yes $(n=374)$ & $344(92.0 \%)$ & $30(8.0 \%)$ & 0.52 & 2.78 & $\mathrm{OR}=1.21$ \\
\hline \multicolumn{6}{|l|}{ Parity } \\
\hline $0(n=222)$ & $207(93.2 \%)$ & $15(6.8 \%)$ & & & 0.30 \\
\hline$\geq 1(n=214)$ & $193(90.2 \%)$ & $21(9.8 \%)$ & 0.37 & 1.30 & $\mathrm{OR}=0.69$ \\
\hline \multicolumn{6}{|l|}{ Previous CS } \\
\hline No $(n=423)$ & $388(91.7 \%)$ & $35(8.3 \%)$ & & & 1.00 \\
\hline Yes $(n=13)$ & $12(92.3 \%)$ & $1(7.7 \%)$ & 0.16 & 1.26 & $O R=1.08$ \\
\hline $\mathrm{BMI}\left(\mathrm{Kg} / \mathrm{m}^{2}\right)$ & $27.81 \pm 3.35$ & $26.99 \pm 3.69$ & -0.34 & 1.97 & 0.17 \\
\hline Pulse rate & $83.9 \pm 5.3$ & $83.4 \pm 5.1$ & -1.32 & 2.27 & 0.60 \\
\hline Temperature $\left({ }^{\circ} \mathrm{C}\right.$ ) & $36.8 \pm 0.2$ & $36.8 \pm 0.3$ & -0.07 & 0.10 & 0.72 \\
\hline \multicolumn{6}{|l|}{ Stage of labour } \\
\hline Latent $(n=68)$ & $61(89.7 \%)$ & $7(10.3 \%)$ & & & 0.63 \\
\hline Active $(n=368)$ & $339(92.1 \%$ & $29(7.9 \%)$ & 0.59 & 2.86 & $\mathrm{OR}=1.31$ \\
\hline \multicolumn{6}{|l|}{ Presentation } \\
\hline Cephalic $(n=429)$ & $393(91.6 \%)$ & $36(8.4 \%)$ & & & 1.00 \\
\hline $\operatorname{Breech}(n=7)$ & $7(100 \%)$ & 0 & 1.06 & 1.12 & $O R=1.09$ \\
\hline \multicolumn{6}{|l|}{ Pregnancy risk } \\
\hline Low risk $(n=354)$ & $325(91.8 \%)$ & $29(8.2 \%)$ & & & 1.00 \\
\hline High risk $(n=82)$ & $75(91.5 \%)$ & $7(8.5 \%)$ & 0.44 & 2.11 & $O R=0.96$ \\
\hline \multicolumn{6}{|c|}{ Interpreted CTG finding } \\
\hline Normal $(n=306)$ & $304(99.3 \%)$ & $2(0.7 \%)$ & & & $<0.001$ \\
\hline Abnormal $(n=130)$ & $96(73.8 \%)$ & $34(26.2 \%)$ & 0.006 & 0.102 & $O R=0.025$ \\
\hline
\end{tabular}

The observed incidences of normal, suspicious and pathological CTG findings obtained in our study is comparable to that obtained by Dwarakanth et al in a study on the efficacy of admission CTG as a risk screening test [8]. These incidences are also comparable to reports from a similar study among parturient with high risk pregnancy [4]. The incidence of pathological CTG finding is also in consonance with that obtained from a study in a similar low resource setting [15]. In an earlier report in Nigeria, from an audit on CTG of women admitted for high risk pregnancies and induction of labour, more than half of the parturient who delivered within one week of the antenatal CTG had non-reactive result [16].

The maternal socio-demographic factors; age, educational status, employment status, history of previous caesarean section and pregnancy risk had no effect on the decisions based on CTG findings. The stage of labour, fetal presentation and the state of fetal membranes had no effect on the decisions that were taken after CTG. Other factors that did not affect these decisions include; maternal BMI, maternal heart rate and temperature, although temperature and heart rate are recognised determinants of FHR on CTG tracings. This finding is so because sufficient confounders have been excluded from the protocol of this study, thus, the CTG reinterpretations can be considered as reliable. The principal determinant of the decision to stop or continue labour in this study was the admission CTG finding $(P<0.001$, OR $=0.025$, $95 \% \mathrm{Cl}=0.006-0.102)$. The decisions from our observations can thus be considered as a consequence of the CTG findings. 
Table 4. Perinatal outcomes associated with decisions based on admission CTG findings

\begin{tabular}{|c|c|c|c|c|c|c|}
\hline \multirow[t]{2}{*}{ Factor } & \multicolumn{2}{|c|}{ Fifth minute APGAR score } & \multirow[t]{2}{*}{$P$-value } & \multirow[t]{2}{*}{$\mathrm{AOR}^{\mathrm{a}}$} & \multicolumn{2}{|c|}{$95 \% \mathrm{Cl}$} \\
\hline & $>7(n=277)$ & $\leq 7(n=159)$ & & & Lower & Upper \\
\hline \multicolumn{7}{|l|}{ Decision to stop labour } \\
\hline $\begin{array}{l}\text { No }(n=400) \\
\text { Yes }(n=36)\end{array}$ & $\begin{array}{l}255(63.8 \%) \\
22(61.1 \%) \\
\end{array}$ & $\begin{array}{l}145(36.2 \%) \\
14(38.9 \%)\end{array}$ & 0.26 & 0.15 & 0.01 & 4.04 \\
\hline \multicolumn{7}{|c|}{ Intrapartum FHR monitoring pattern $(n=400)$} \\
\hline $\begin{array}{l}\text { Intermittent }(n=300) \\
\text { EFM }(n=100)\end{array}$ & $\begin{array}{l}194(64.7 \%) \\
61(61.0 \%)\end{array}$ & $\begin{array}{l}106(35.3 \%) \\
39(39.0 \%)\end{array}$ & 0.66 & 0.84 & 0.39 & 1.84 \\
\hline \multicolumn{7}{|l|}{ Admission CTG finding } \\
\hline $\begin{array}{l}\text { Normal }(n=306) \\
\text { Abnormal }(n=130)\end{array}$ & $\begin{array}{l}199(45.6 \%) \\
78(60.0 \%) \\
\end{array}$ & $\begin{array}{l}107(35.0 \%) \\
52(40.0 \%)\end{array}$ & 0.55 & 0.77 & 0.38 & 1.69 \\
\hline \multirow[t]{2}{*}{ Factor } & \multicolumn{2}{|c|}{ Suspected fetal distress } & P-value & AOR $^{b}$ & \multicolumn{2}{|c|}{$95 \% \mathrm{Cl}$} \\
\hline & No & Yes & & & Lower & Upper \\
\hline $\begin{array}{l}\text { Intrapartum FHR monitoring } \\
\text { pattern }(n=400)\end{array}$ & $\mathrm{n}=388$ & $\mathrm{n}=12$ & & & & \\
\hline $\begin{array}{l}\text { Intermittent }(n=300) \\
\text { EFM }(n=100)\end{array}$ & $\begin{array}{l}291(97.0 \%) \\
97(97.0 \%)\end{array}$ & $\begin{array}{l}9(3.0 \%) \\
3(3.0 \%)\end{array}$ & 0.42 & 2.12 & 0.39 & 13.31 \\
\hline Admission CTG finding & $n=416$ & $\mathrm{n}=\mathbf{2 0}$ & & & & \\
\hline $\begin{array}{l}\text { Normal }(n=306) \\
\text { Abnormal }(n=130)\end{array}$ & $\begin{array}{l}298(97.4 \%) \\
118((90.8 \%)\end{array}$ & $\begin{array}{l}8(2.6 \%) \\
12(9.2 \%)\end{array}$ & 0.91 & 0.91 & 0.17 & 4.98 \\
\hline
\end{tabular}

Occurrence of CTG abnormality did not correlate significantly with the clinical suspicion of fetal distress, this is in consonance with a report by Desai et al where CTG patterns did not correlate with occurrence of fetal distress, but contrasts reports from India and Nepal where Admission CTG findings were found to correlate with the 5minute APGAR scores $[4,8,17]$. Some other studies done in developed countries where CTG use is commoner, reported limited association between CTG and fetal outcomes; false positive predictive rates for fetal distress as high as $99 \%$ were found $[18,19]$. The decision to continue or stop labour did not affect the neonatal five minute APGAR scores significantly, similarly, a comparison between intermittent auscultation with the fetal stethoscope and continuous EFM revealed no difference in occurrence of fetal distress and occurrence of neonatal APGAR score $\leq 7$. The lack of a strong link between abnormal CTG (suspicious and pathological) and fetal acidosis has been earlier reported [20].

Occurrence of maternal near miss and complications of labour did not depend on the decision to continue or stop labour; in addition, labour managed with intermittent FHR auscultation was not significantly different from that in which continuous EFM was done with respect to occurrence of maternal near miss or complications of labour. A higher rate of vaginal deliveries were noted among women who had normal CTG findings, this is comparable to what was obtained in Nepal [4]. A higher rate of operative deliveries occurred among parturient who had their labour stopped abruptly because of 'abnormal' CTG findings, this finding is in consonance with earlier reports from studies done in India $[8,21,22]$. Caution in interpreting CTG findings has been advised and fetal scalp blood sampling for fetal $\mathrm{pH}$ to determine true acidosis has been advocated whenever critical decisions based on CTG findings are contemplated $[4,12,13]$. Clinical decisions based on non-uniform interpretation of CTG findings have earlier been hypothesized as important determinants of the high rate of unnecessary operative obstetric interventions related to CTG use [12].

The pregnancy risk estimation at admission was however, a significant determinant of maternal outcome as low risk pregnancies had significantly lower rates of MNM, operative deliveries and maternal complications of labour. In contrast, the use of admission CTG in some studies found a correlation between CTG and obstetric risk as screening tools $[4,8]$. 
Table 5. Maternal outcomes associated with decisions based on admission CTG

\begin{tabular}{|c|c|c|c|c|c|c|}
\hline \multirow[t]{2}{*}{ Factor } & \multicolumn{2}{|c|}{$\begin{array}{l}\text { Occurrence of Maternal } \\
\text { Near Miss (MNM) }\end{array}$} & \multirow[t]{2}{*}{$P$-value } & \multirow[t]{2}{*}{$\mathrm{AOR}^{\mathrm{a}}$} & \multicolumn{2}{|c|}{$95 \% \mathrm{Cl}$} \\
\hline & No & Yes & & & Lower & Upper \\
\hline \multicolumn{7}{|c|}{ Decision to stop labour } \\
\hline $\mathrm{No}(\mathrm{n}=400)$ & $360(90.0 \%)$ & $40(10.0 \%)$ & 0.98 & 0.72 & 0.30 & 1.71 \\
\hline Yes $(n=36)$ & $31(86.1 \%)$ & $5(13.9 \%)$ & & & & \\
\hline \multicolumn{7}{|c|}{ Intrapartum FHR monitoring pattern $(n=400)$} \\
\hline Intermittent $(n=300)$ & $282(94.0 \%)$ & $18(6.0 \%)$ & 0.19 & 0.25 & 0.03 & 1.96 \\
\hline $\operatorname{EFM}(n=100)$ & $78(78.0 \%)$ & $22(22.0 \%)$ & & & & \\
\hline \multicolumn{7}{|c|}{ Admission CTG finding } \\
\hline Normal $(n=306)$ & $275(89.9 \%)$ & $31(10.1 \%)$ & 0.38 & 0.44 & 0.07 & 2.76 \\
\hline Abnormal $(n=130)$ & $116(89.2 \%)$ & $14(10.8 \%)$ & & & & \\
\hline \multicolumn{7}{|l|}{ Pregnancy risk } \\
\hline Low risk $(n=354)$ & $351(99.2 \%)$ & $3(0.8 \%)$ & $<0.001$ & 0.002 & 0.000 & 0.032 \\
\hline High risk $(n=82)$ & $40(48.8 \%)$ & $42(51.2 \%)$ & & & & \\
\hline \multirow[t]{2}{*}{ Factor } & \multicolumn{2}{|c|}{$\begin{array}{l}\text { Occurrence of maternal } \\
\text { complication of labour } \\
(n=400)^{*}\end{array}$} & $P$-value & $\mathrm{AOR}^{\mathrm{b}}$ & \multicolumn{2}{|c|}{$95 \% \mathrm{Cl}$} \\
\hline & No(n=330) & Yes(n=70) & & & Lower & Upper \\
\hline \multicolumn{7}{|c|}{ Intrapartum FHR monitoring pattern $(n=400)$} \\
\hline Intermittent $(n=300)$ & $256(85.3 \%)$ & $44(14.7 \%)$ & 0.34 & 0.66 & 0.28 & 1.56 \\
\hline $\operatorname{EFM}(n=100)$ & $74(74.0 \%)$ & $26(26.0 \%)$ & & & & \\
\hline \multicolumn{7}{|c|}{ Admission CTG finding } \\
\hline Normal $(n=303)$ & $250(82.5 \%)$ & $53(17.5 \%)$ & 0.38 & 1.47 & 0.62 & 3.49 \\
\hline Abnormal $(n=97)$ & $80(82.5 \%)$ & $17(17.5 \%)$ & & & & \\
\hline \multicolumn{7}{|l|}{ Pregnancy risk } \\
\hline Low risk $(\mathrm{n}=326)$ & $289(88.7 \%)$ & $37(11.3 \%)$ & $<0.001$ & 0.142 & 0.069 & 0.292 \\
\hline High risk $(n=74)$ & $41(54.1 \%)$ & $33(44.6 \%)$ & & & & \\
\hline
\end{tabular}

Table 6. Comparison of mode of delivery outcomes between pregnancy risk and admission CTG findings and decisions

\begin{tabular}{|c|c|c|c|c|c|c|}
\hline \multirow[t]{2}{*}{ Factor } & \multicolumn{2}{|c|}{ Mode of delivery } & \multirow[t]{2}{*}{$P$-value } & \multirow[t]{2}{*}{$\mathrm{AOR}^{\mathrm{a}}$} & \multicolumn{2}{|c|}{$95 \% \mathrm{Cl}$} \\
\hline & Non-operative & Operative & & & Lower & Upper \\
\hline \multicolumn{7}{|c|}{ Decision to stop labour } \\
\hline No $(n=400)$ & $335(83.8 \%)$ & $65(16.2 \%)$ & $<0.001$ & 0.202 & 0.153 & 0.265 \\
\hline Yes $(n=36)$ & $7(19.4 \%)$ & $29(80.6 \%)$ & & & & \\
\hline \multicolumn{7}{|c|}{ Intrapartum FHR monitoring pattern $(n=400)$} \\
\hline Intermittent $(n=300)$ & $261(87.0 \%)$ & $39(13.0 \%)$ & 0.22 & 1.92 & 0.68 & 5.43 \\
\hline $\operatorname{EFM}(n=100)$ & $72(72.0 \%)$ & $28(28.0 \%)$ & & & & \\
\hline \multicolumn{7}{|l|}{ Pregnancy Risk } \\
\hline Low risk $(n=354)$ & $314(88.7 \%)$ & $40(11.3 \%)$ & $<0.001$ & 0.015 & 0.005 & 0.043 \\
\hline High risk $(n=82)$ & $28(34.1 \%)$ & $54(65.9 \%)$ & & & & \\
\hline
\end{tabular}

The failure of admission CTG or continuous intrapartum CTG to have a significant positive impact on perinatal or maternal outcome has led to calls against its routine use in labour either as a screening tool or for monitoring, especially when the huge expenses lost to litigation related to labour management were considered $[9,10,23]$. 
Our inability to compare obstetric risk-based triage with admission CTG as predictors of outcome is the main limitation of this study; a prospective randomized control trial may be useful to make this inference. The inability to access the long term impact of the CTG-based decisions on childhood development is also a limitation, a cohort study design may be necessary for this assessment. The major strength of this study is the large number of available CTG print-outs for analysis and the retrospective nature of the design, with reduced potential for bias.

\section{CONCLUSION}

Decision for immediate delivery based on CTG was associated with a high rate of operative deliveries, without any significant improvement in fetal or maternal outcomes. Intermittent auscultation was comparable with EFM with regards to maternal or fetal outcomes. Uniform and user-friendly guidelines should be used for CTG protocol development. Electronic fetal monitoring in labour should be restricted to pregnancies adjudged as high risk based on obstetric factors and CTG should be used as an adjunctive triaging instrument.

\section{ETHICAL APPROVAL}

Ethical clearance was obtained for this study from the Babcock University Health and Research Ethics Committee (BUHREC), protocol number, BUHREC 169/19, before commencement of data extraction.

\section{ACKNOWLEDGEMENTS}

The authors express sincere gratitude to the nursing staff and the staff of the medical records department for their support and cooperation towards retrieval of patient files, delivery register and other relevant hospital records, that contributed to the success of this audit.

\section{COMPETING INTERESTS}

Authors have declared that no competing interests exist.

\section{REFERENCES}

1. Van HP. Developments in CTG analysis. Bailleres Clin Obstet Gynaecol. 1996;10: 185-207.

2. Devane D, Lalor JG, Daly S, McGuire W, Cuthbert A, Smith V. Cardiotocography versus intermittent auscultation of fetal heart on admission to labour ward for assessment of fetal well-being. Cochrane database of Systematic Reviews. (1). Art: CD 005122. Pub.5. John Wiley and Sons. Ltd; 2017.

DOI: 10.1002/14651858. CD005122

3. Das V, Katiyar N, Malik GK. Role of admission test. J Obstet Gynecol India. 2001;51(1):48-50.

4. Thapa J, Sah R. Admission cardiotocography in high risk pregnancies. NJOG. 2017;23(1):50-54.

5. National institute for health and care excellence. Intrapartum Care: NICE Guideline CG190; 2017.

Available:https://www.nice.org.uk/guidance lcg190

[Accessed on 23/04/2018]

6. Shriver EK. What is a high-risk pregnancy? Healthy pregnancies, healthy children and optimal lives. National Institute of Child Health and Human Development.

Available:https://www.nichd.nih.gov/health/ topics/pregnancy/conditionsinfo/high-risk [Accessed on 26/09/2019]

7. Say L, Souza JP, Pattinson RC. WHO working group on maternal mortality and morbidity classification. Maternal near miss-towards a standard tool for monitoring quality of maternal health care. Best Pract Res Clin Obstet Gynaecol. 2009;23:287-296.

8. Dwarakanath L, Lakshmikantha G, Chaitra SK. Efficacy of admission cardiotocography (admission test) to predict obstetric outcome. J. Evol Med Dental Sci. 2013;2(5):418-423.

9. Sartwelle TP. Electronic fetal monitoring: A defense lawyer's view. Rev Obstet Gynecol. 2012;5(3/4):e121-e125.

10. Alfirevic Z, Devane D, Gyte GM. Continuous Cardiotocography (CTG) as a form of Electronic Fetal Monitoring (EFM) for fetal assessment during labour. Cochrane Database of Systematic Reviews. 2013;31(5):CD006066.

11. Vintzileos AM, Nochimson DJ, Guzman ER, Knuppel RA, Lake M, Schifrin BS. Intermittent electronic fetal heart rate monitoring versus intermittent auscultation: A meta-analysis. Obstet Gynecol. 1995;85: 149-55.

12. O'Mahony F, Hofmeyr GJ, Menon V. Choice of instruments for assisted vaginal 
delivery. Cochrane Database Syst Rev. 2010;10(11):CD005455.

13. Ayres-de-Campos D, Bernardes J, CostaPereira A, Pereira-Leite L. Inconsistencies in classification by experts of cardiotocograms and subsequent clinical decision. BJOG. 1999;106:1307-10.

14. Blackwell SC, Grobman WA, Antoniewicz L, Hutchinson M, Gyamfi-Bannerman C. Interobserver and intraobserver reliability of the $\mathrm{NICH}$ 3-tier fetal heart rate interpretation system. Am J Obstet Gynecol. 2011;205:378.e1-5.

15. Rahman H, Renjhen P, Dutta S. Reliability of admission cardiotocography for intrapartum monitoring in low resource setting. Niger Med J. 2012;53(3): 145-149.

16. Fawole AO, Sotiloye OS, Oladimeji AO, Alao MO, Hunyinbo KI, Sadoh EA, Otolorin EO. Antenatal cardiotocography: Experience in a Nigerian tertiary hospital. Nigerian Postgraduate Medical Journal. 2008;15:19-23.

17. Desai D, Maitra N, Patel P. Fetal heart rate patterns in patients with thick meconium staining of amniotic fluid and its association with perinatal outcome. Int $\mathrm{J}$
Reprod Contracept Obstet Gynecol. 2017; 6(3);1030-1035.

18. Nelson KB, Dambrosia JM, Ting TY, Grether JK. Uncertain value of electronic fetal monitoring in predicting cerebral palsy. N Engl J Med. 1996;334:613-618.

19. Barrett JF, Jarvis GJ, Macdonald HN, et al. Inconsistencies in clinical decisions in obstetrics. Lancet. 1990;336:549-551.

20. Spencer JA. Clinical overview of cardiotocography. $\mathrm{Br} \mathrm{J}$ Obstet Gynaecol. 1993;100(9):4-7.

21. Kamal B, Deepika D, Padmaja V. Admission test as predictor of fetal outcome. J Obstet and Gynaec of India. 1999;49(2):36-37.

22. Ingemarsson I, Arulkumaran $S$, Ingemarsson E, Tambyraja RI, Ratnam SS. Admission test a screening test for fetal distress in labour. Obstet Gynecol. 1986;68(6):800-806.

23. Ayres-de-Campos D, Bernardes J, Marsal K, Nickelsen C, Makarainen L, Banfield P, et al. Can the reproducibility of fetal heart rate baseline estimation be improved? Eur J Obstet Gynecol Reprod Biol. 2004;112: 49-54.

(c) 2019 Imaralu et al.; This is an Open Access article distributed under the terms of the Creative Commons Attribution License (http://creativecommons.org/licenses/by/4.0), which permits unrestricted use, distribution, and reproduction in any medium, provided the original work is properly cited.

Peer-review history:

The peer review history for this paper can be accessed here: http://www.sdiarticle4.com/review-history/51777 